
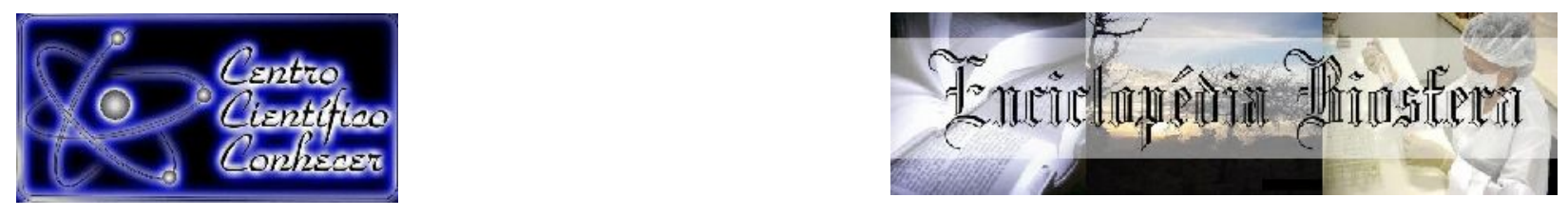

\title{
EFICÁCIA DE EXTRATOS VEGETAIS DE Punica granatum L. NO CONTROLE in vitro DE OVOS E ADULTOS DE Fasciola hepatica
}

Maria Larissa Bitencourt Vidal'; Isabella Vilhena Freire Martins ${ }^{1}$; Jankerle Neves Boeloni1; Thays de Carvalho Amorim Bolzan1; Juliana Aparecida Severi²

1Departamento de Medicina Veterinária- Centro de Ciências Agrárias e EngenhariasCCAE- Universidade Federal do Espírito Santo- UFES, Alegre, ES- Brasil.

2Departamento de Farmácia- Centro de Ciências Exatas, Naturais e da Saúde CCENS- Universidade Federal do Espírito Santo- UFES, Alegre,ES-Brasil.

Email: larissabvidal@gmail.com

Recebido em: 06/04/2019 - Aprovado em: 10/06/2019 - Publicado em: 30/06/2019

DOI: 10.18677/EnciBio_2019A61

\begin{abstract}
O objetivo do presente trabalho foi avaliar a eficácia do extrato da casca do fruto de Punica granatum L. no controle "in vitro" de ovos e adultos de Fasciola hepatica. A determinação da composição química foi a partir da avaliação fitoquímica, que é uma técnica qualitativa que confirma a presença ou não de compostos fenólicos, flavonóides, taninos, cumarinas, terpenos, glicosídeos cardiotônicos, alcalóides e saponinas. O ensaio em ovos foi realizado em triplicata a partir de soluções de $0,1 \%$, $0,25 \%$ e $0,5 \%$ do extrato e sobre os adultos foi utilizado um parasito por placa em triplicata semelhante à análise dos ovos e avaliado nas horas $0 \mathrm{~h}, 1 \mathrm{~h}, 3 \mathrm{~h}, 12 \mathrm{~h}$ e $15 \mathrm{~h}$, observando a morte (ou não) dos espécimes, por avaliação da motilidade. A partir dos ensaios foi possível evidenciar atividade fasciolicida da casca do fruto de $P$. granatum L. em todas as concentrações nos ovos e nos adultos a de $0,1 \%$ e $0,5 \%$ tiveram maior atividade, estando associadas à presença dos compostos metabólicos como taninos, flavonóides e compostos fenólicos nos extratos vegetais utilizados. Conclui-se que todas as concentrações, porém com efeito superior à de $0,5 \% \mathrm{com}$ potencial sobre os ovos e adultos frente à $F$. hepatica.
\end{abstract}

RESUMO

PALAVRAS-CHAVE: fasciolose; plantas; tratamento

\section{EFFECTIVENESS OF VEGETABLE EXTRACTS OF Punica granatum L. IN CONTROL in vitro OF EGGS AND ADULTS OF Fasciola hepatica}

\begin{abstract}
The objective of the present work was to evaluate the efficacy of the extract of the bark of the fruit of Punica granatum $L$. in the in vitro control of eggs and adults of $F$. hepatica. The determination of the chemical composition was based on phytochemical evaluation, which is a qualitative technique that confirms the presence or absence of phenolic compounds, flavonoids, tannins, coumarins, terpenes, cardiotonic glycosides, alkaloids and saponins. The egg assay was performed in triplicate from solutions of $0.1 \%, 0.25 \%$ and $0.5 \%$ of the extract and on the adults a parasite per plate was used in triplicate similar to
\end{abstract}


the analysis of the eggs and evaluated in the hours $0 \mathrm{~h}, 1 \mathrm{~h}, 3 \mathrm{~h}, 12 \mathrm{~h}$ and $15 \mathrm{~h}$, observing the death (or not) of the specimens, by evaluation of the motility. From the tests, it was possible to evidence the fasciolicidal activity of $P$. granatum $L$. fruit peel at all concentrations in eggs and in adults at $0.1 \%$ and $0.5 \%$ had greater activity, being associated with the presence of metabolic compounds such as tannins, flavonoids and phenolic compounds in the plant extracts used. It was concluded that all concentrations, but with effect higher than $0.5 \%$ with potential on eggs and adults compared to Fasciola hepatica.

KEYWORDS: fasciolosis; plants; treatment

\section{INTRODUÇÃO}

O parasito Fasciola hepatica (Linnaeus, 1758), é um dos helmintos mais importantes que parasitam animais domésticos e selvagens no Brasil com altas taxas de mortalidade e morbidade de animais afetados. Fasciolose, a doença provocada por $F$. hepatica, com distribuição mundial, compreende uma zoonose relevante na produção animal, com perdas econômicas principalmente em bovinos e ovinos, e também afeta a saúde pública (JACOBS et al., 2015, ANDREANI et al., 2017, QUEVEDO et al., 2018).

O tratamento químico é uma das principais formas de controle de animais parasitados. Entretanto, os riscos da resistência anti-helmíntica, os resíduos químicos gerados com a administração dos medicamentos, a disponibilidade e alto custo têm despertado a necessidade de desenvolvimento de métodos alternativos de controle. O controle biológico frente aos invertebrados que participam do ciclo de vida dos parasitos e atuando frente aos próprios parasitos, a partir da utilização de fungos, extratos e óleos oriundos de plantas medicinais, entre outros, têm sido estudados em diferentes partes do mundo (ALVAREZ-MERCADO et al., 2015, TUNHOLI et al., 2017).

A utilização de extratos de plantas é considerada uma alternativa bastante promissora no tratamento de afecções parasitárias, mediante a disponibilidade e sustentabilidade e por não gerar resíduos nos produtos de origem animal. Atualmente recomendam-se medidas estratégicas preventivas associadas ao tratamento dos indivíduos infectados em programas de controle, minimizando a taxa de contaminação ambiental. Por estas e outras razões, medidas alternativas de controle vêm sendo desenvolvidas, e algumas são baseadas na possibilidade do uso de plantas ou de seus derivados como agentes moluscicidas, além da utilização de micro-organismos patogênicos (TUNHOLI et al., 2017).

Apesar dos extratos de plantas representarem um potencial alternativo para o controle eficaz da fasciolose poucos estudos têm sido desenvolvidos, sendo necessária a realização de novas pesquisas para determinar seu potencial (ALVAREZ-MERCADO et al., 2015). No entanto, os extratos de $P$. granatum L., já foram testados e inúmeros estudos têm comprovado propriedades anti-helmínticas relacionadas a esta espécie vegetal (LALHMINGCHHUANMAWII, et al., 2014).

Entretanto, não foram encontrados relatos sobre potencial da atividade fasciolicida em ovos e adultos de $F$. hepatica de extratos de $P$. granatum $L$, com isso o objetivo deste trabalho foi avaliar pela primeira vez a eficácia in vitro do extrato da casca do fruto de $P$. granatum L. sobre ovos e adultos de $F$. hepatica. 


\section{MATERIAL E MÉTODOS}

O experimento foi realizado de março a julho de 2017. A coleta dos helmintos adultos e os ovos de $F$. hepatica, utilizados para o experimento foi realizada em matadouro-frigorífico da região Sul do Estado do Espírito Santo, com autorização prévia, sendo encaminhados para o Laboratório de Parasitologia da UFES (Universidade Federal do Espírito Santo).

Os extratos de $P$. granatum utilizados para avaliar a possível atividade fasciolicida sobre ovos e adultos foram produzidos no Laboratório de Produção Farmacêutica do Centro de Ciências Exatas, Naturais e da Saúde da Universidade Federal do Espírito Santo. O material vegetal utilizado neste trabalho foi selecionado a partir de extratos vegetais disponíveis do acervo do Laboratório de Produção Farmacêutica, sendo selecionado o extrato de $P$. granatum $L$, utilizado nas concentrações de $0,1 \%, 0,25 \%$ e $0,5 \%$. Em seguida, os ensaios biológicos foram conduzidos nos Laboratórios de Parasitologia e Patologia Animal, ambos localizados no Hospital Veterinário no Centro de Ciências Agrárias e Engenharias da Universidade Federal do Espírito Santo.

A avaliação da composição química do extrato nas diferentes concentrações $(0,1 \%, 0,25 \%$ e $0,5 \%)$ foi realizada com base nos protocolos propostos por Matos (2009). Os testes realizados tiveram por objetivo confirmar a presença ou não de compostos fenólicos, flavonóides, taninos, cumarinas, terpenos, glicosídeos cardiotônicos, alcalóides e saponinas.

Os ensaios de avaliação da atividade fasciolicida sobre os ovos foram inicialmente realizados com os extratos brutos em solução aquosa, obtendo soluções a $0,1 \%, 0,25 \%$ e $0,5 \%$, em quantidade compatível para a execução dos ensaios biológicos em triplicata.

A avaliação da atividade fasciolicida sobre os ovos do helminto foi realizada conforme a metodologia descrita por Fairweather et al. (2012). Para separação do máximo de bile dos ovos, estes foram submetidos à sedimentação, após a sedimentação, os ovos foram contabilizados em placa de petri. Assim que foi determinada a quantidade de ovos por amostra, definido por 100 ovos, estes foram depositados em tubos tipo Falcon de $50 \mathrm{~mL}$. Foram adicionados $3 \mathrm{~mL}$ do extrato de Punica granatum L. em cada concentração $(0,1 \% ; 0,25 \%$ e $0,5 \%)$ nos tubos juntamente com os ovos. Para controle positivo, utilizou-se albendazol na concentração de $0,5 \%$ e para controle negativo utilizou-se água destilada.

Os tubos foram embalados em papel alumínio para que os ovos não tivessem contato com a luz ambiente. Estes foram encaminhados para uma estufa de demanda de oxigênio tipo B.O.D. a uma temperatura controlada de $25^{\circ} \mathrm{C}$, avaliados em 14 dias, não havendo eclosão de miracídios, permaneceram por 21 dias. Passados 21 dias, os ovos foram retirados da B.O.D. e expostos a luz incandescente de $100 \mathrm{~W}$, por três horas, para realização da análise (FAIRWEATHER et al., 2012). Os ovos foram analisados e contados a cada hora, durante três horas, com o auxílio de microscópio estereoscópio no aumento de duas vezes.

Ao fim das análises de eclosão, os ovos foram pipetados e depositados em lâminas, juntamente com água, e cobertos com lamínula, para que fossem avaliados morfologicamente. Foram avaliadas pelo menos duas lâminas, com média de cinco ovos em cada, observando tamanho, conservação da parede externa, formação ou não de miracídios e abertura ou não do opérculo, por meio de microscópio óptico Olympus CX41, usando as objetivas de 40x e 100x. 
Os ensaios de avaliação da atividade fasciolicida sobre os parasitos adultos foram semelhantes aqueles realizados para os ovos e baseado na metodologia Fairweather et al. (2012) a partir de extratos brutos diluídos em solução aquosa para obtenção das soluções nas concentrações de $0,1 \%, 0,25 \%$ e $0,5 \%$.

Após a coleta dos parasitos adultos, imediatamente foram montados os cinco grupos experimentais, representados pelos grupos expostos aos extratos vegetais nas concentrações de $0,1 \%, 0,25 \%, 0,5 \%$, estabelecidas por trabalhos realizados anteriormente, controle positivo e controle negativo. Cada grupo experimental foi constituído por um parasito adulto, devidamente acondicionado em placa de Petri de $35 \times 10 \mathrm{~mm}$ em triplicata, contendo $10 \mathrm{~mL}$ de meio RPMI 1640. Aos grupos expostos a ação dos extratos aplicou-se mais $10 \mathrm{~mL}$ do extrato de $P$. granatum $L$. nas concentrações de $0,1 \%, 0,25 \%$ e $0,5 \%$. Ao grupo controle negativo não foi adicionado nenhuma solução além do meio RPMI 1640, e o controle positivo foi constituído pela adição de $10 \mathrm{~mL}$ de albendazol à $0,5 \%$.

Em seguida as amostras foram avaliadas nas horas $0 \mathrm{~h}, 1 \mathrm{~h}, 3 \mathrm{~h}, 12 \mathrm{~h}$ e $15 \mathrm{~h}$, observando a morte (ou não). A motilidade foi o parâmetro utilizado para avaliar a viabilidade dos parasitos durante o período de análise. A fim de realizar a avaliação histológica dos parasitos frente aos extratos, ao final da avaliação (15h) coletaramse todos os exemplares de parasitos de cada grupo experimental.

Depois de realizadas as avaliações das atividades fasciolicidas dos extratos, os parasitos foram fixados em Bouin (Biofix®) por 24 horas e após esse período foram fixados em formalina $10 \%$ por uma hora. Posteriormente foram realizadas secções transversais da $F$. hepatica, sendo dividida em três porções (terços inicial, médio e final) para melhor avaliar as áreas de tegumento e dos órgãos reprodutivos do parasito.

Foram realizados cortes de quatro micrômetros de espessura e em seguida corados por hematoxilina-eosina. As lâminas foram examinadas utilizando os índices propostos pela World Association for the Advancement of Veterinary Parasitology (W.A.A.V.P) por microscopia óptica de luz para determinar as possíveis modificações externas e internas induzidas pela ação dos extratos em microscópio óptico Olympus CX41 de luz. As alterações encontradas foram descritas e a captura das imagens foi realizada pela câmera Sony DSC-W610.

Para determinação da eficácia do extrato vegetal sobre os ovos, foram realizados cálculos de eficácia, utilizando a seguinte fórmula: \% eficácia = (média do grupo controle negativo - média do grupo tratado com extrato / média do grupo controle negativo) $\times 100$. Também foi realizada análise descritiva da microscopia dos ovos e da histologia dos exemplares adultos.

Para os resultados das três repetições de cada extrato vegetal dos adultos, nas horas um, dois e três foram comparados por meio de cálculo estatístico realizado por delineamento inteiramente casualizado. Os dados foram submetidos à análise de variância e as médias comparadas pelo teste de Tukey. Diferenças foram consideradas significativas se $p<0,05$.

\section{RESULTADOS E DISCUSSÃO}

Nos testes realizados na avaliação fitoquímica foi possível caracterizar as principais classes de metabólitos presentes no extrato estudado. Foram descritas pela avaliação fitoquímica (quadro 1) da casca do fruto de P.granatum L. observa-se a presença de compostos fenólicos, flavonóides e taninos. 
QUADRO 1. Análise da composição fitoquímica do extrato de Punica granatum L. com as reações de identificação da presença ou ausência da classe dos compostos testados com o extrato bruto da casca do fruto.

Classe de compostos

Ensaios realizados

Resultado observado

Compostos fenólicos

$\mathrm{FeCl} 35 \%$

Positivo

Flavonóides

$\mathrm{AlCl} 35 \%$

positivo

Pacheco

positivo

Shinoda

Pew

positivo

Taubouk

negativo

positivo

Taninos

Gelatina 2\%

positivo

$\mathrm{Pb}(\mathrm{AcO})$

negativo

$\mathrm{FeCl} 31 \%$

positivo

$\mathrm{Cu}(\mathrm{AcO}) 2$

negativo

Cumarinas

$\mathrm{KOH}$

Negativo

Terpenos

Keller

negativo

Pesez

negativo

Libermann-Buchard

negativo

Saponinas

Formação de espuma estável

negativo

Atividade hemolítica

negativo

Alcaloides

Bertrand

negativo

Wagner

Sonnenschein

Dragendorff

Mayer

negativo

negativo

negativo

negativo

FONTE: VIDAL, (2017).

De acordo com Nascimento Junior et al. (2016) as cascas dos frutos apresentaram fitocomplexos como, flavonóides, ácido elágico e punicalagina, corroborando com os resultados apresentados no presente estudo. Ademais, Menezes et al. (2008) descreveram a presença de frações de polifenóis em $P$. granatum L. ricas em flavonóides com propriedades em células cancerígenas e atividade antiangiogênica tanto in vitro, quanto in vivo. Além disso, a casca da romã contém aproximadamente $20 \%$ de taninos, sendo rica em taninos hidrolisáveis, incluindo punicalina, punicalagina, granatinas A e B, 
galagildilactona, casuarinina, pedunculagina, telimagrandina I e corilagina. A punicalagina, principalmente, é o composto responsável pela atividade antioxidante da $P$. granatum L.

Dentre os compostos identificados nas análises fitoquímicas do extrato de $P$. granatum, taninos e flavonóides têm demonstrado eficácia contra ampla diversidade de parasitos. Estudos relatam efeito anti-helmíntico de plantas como Artemisia mexicana, Mentha piperita, Achillea millefolium, Allium sativum, Piper nigrum, Caricapapaya, todas apresentando taninos e flavonoides, contra $F$. hepatica (ALVAREZ-MERCADO et al., 2015).

$\mathrm{Na}$ atividade fasciolicida nos ovos, os extratos vegetais nas concentrações de $0,1 \%, 0,25 \%$ e $0,5 \%$ e o albendazol $0,5 \%$ foram determinantes na inibição da eclosão miracidial. Entretanto, o extrato na concentração de $0,1 \%$ não impediu a eclosão em todas as amostras. No controle negativo não ocorreu inibição da eclosão das larvas.

Os extratos foram analisados utilizando o cálculo de eficácia (Tabela 1). Aqueles que demonstraram ação entre $80-90 \%$ foram considerados efetivos, como 0 extrato de $P$. granatum L. $0,5 \%$, obtendo valor de $83,9 \%$ de eficácia, e o albendazol 0,5\%, com eficácia de $86,53 \%$. Já os inferiores a $80 \%$ foram considerados como não efetivos, sendo representados pelos extratos nas concentrações de $0,1 \%$ e $0,25 \%$, com $45,5 \%$ e $56,73 \%$ de eficácia, respectivamente.

TABELA 1. Avaliação quantitativa dos ovos de Fasciola hepatica após incubação por 21 dias com extrato da casca do fruto de Punica granatum L. em 3 horas de observação e a porcentagem de eficácia na hora 3.

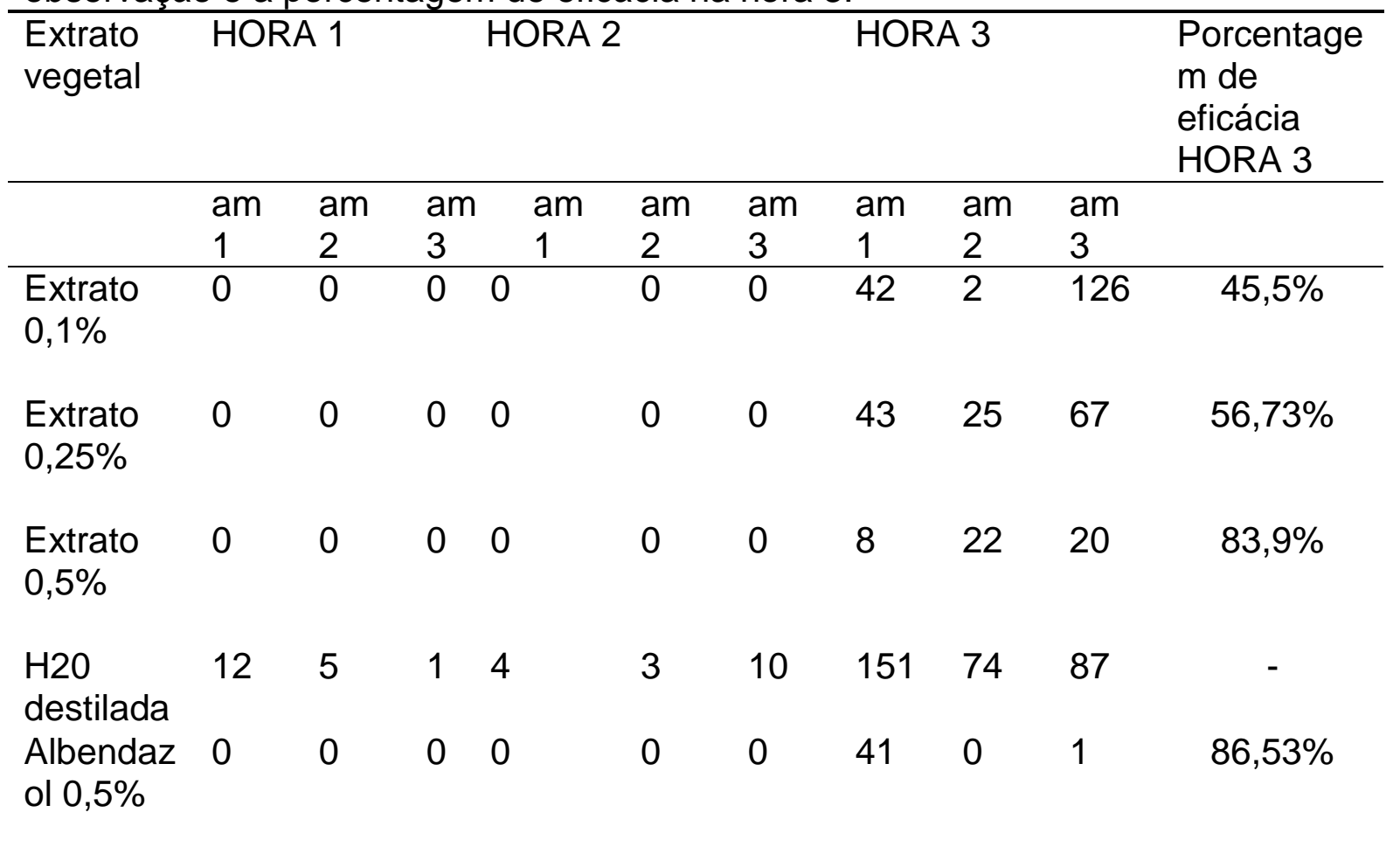

$\mathrm{Am}=$ amostra

Pela avaliação da eficácia sobre os ovos, sugere-se atividade fasciolicida apenas para o extrato de $P$. granatum $L$. na concentração de $0,5 \%$ descrito pela classificação proposta e o albendazol $0,5 \%$, 
considerado princípio ativo químico de eleição utilizado no Brasil frente ao parasito. Os demais apresentaram baixa atividade, $0,1 \%$ e $0,25 \%$ sendo descritos como não efetivos.

$\mathrm{Na}$ análise microscópica dos ovos, a água não impediu a eclosão dos miracídios dos ovos de $F$. hepatica, fazendo desta forma que os opérculos ficassem abertos, como observado na figura 1 A e B. Coles e Stafford (2001) descreveram que o albendazol apresentou excelentes atividades contra os ovos e as formas maduras de $F$. hepatica com resultados de eficácia clínica significativa. Rinaldi et al. (2009) observaram que a ação deste anti-helmíntico está intimamente relacionada a sua capacidade de penetração na casca do ovo, acumulando em seu interior, comprometendo dessa maneira o processo de embriogênese do helminto, impossibilitando a formação ou a eclosão dos miracídios.

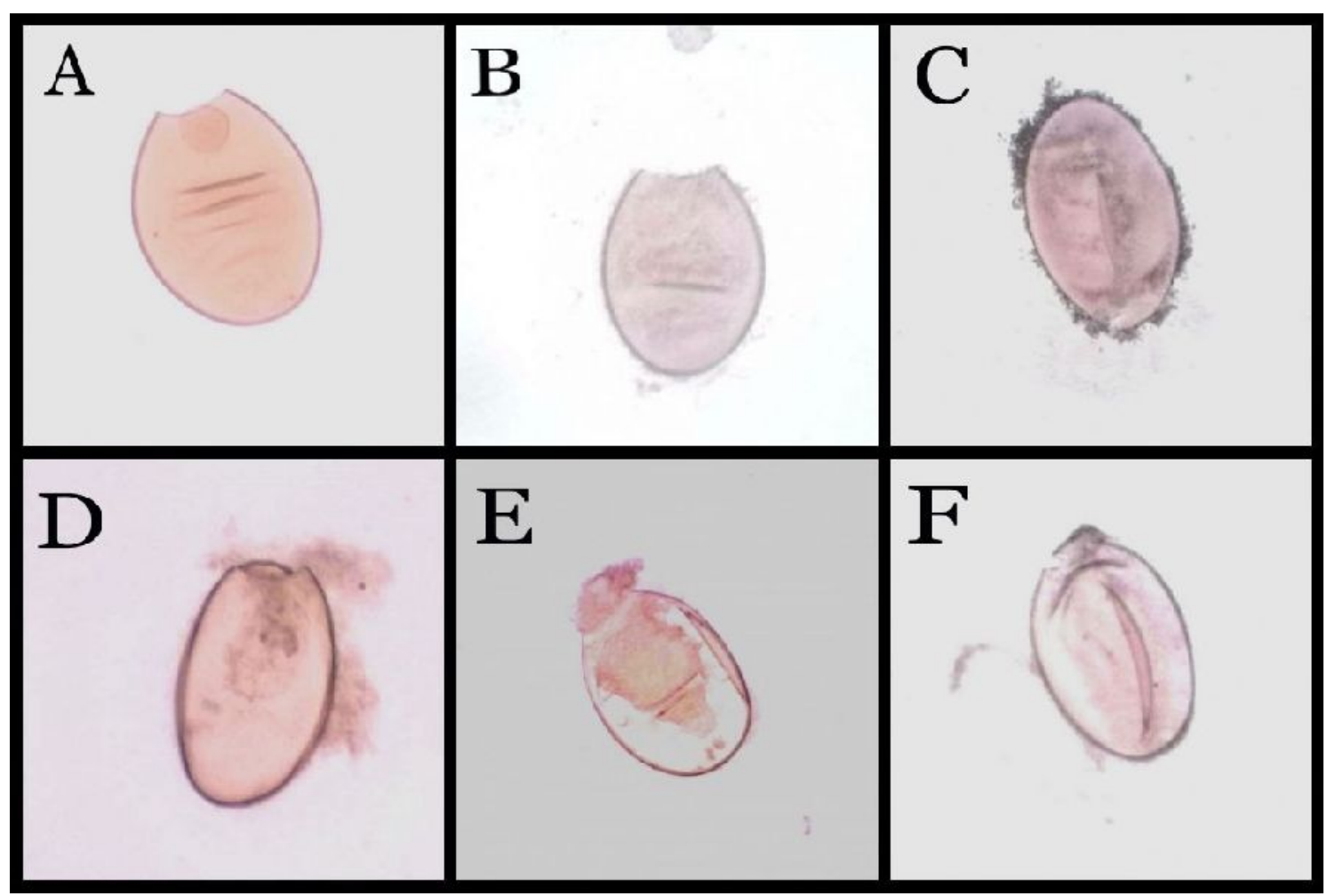

FIGURA 1. Fotomicrografia registrados pelo programa Dino-Eye no aumento de 100x de ovos de Fasciola hepatica, onde A e B representam ovo sob efeito da água; C submetido ao albendazol 0,5\%; D, E e F, sob ação do extrato da casca do fruto de Punica granatum L. na concentração de $0,1 \%, 0,25 \%$ e $0,5 \%$, respectivamente. FONTE: VIDAL,( 2017).

O controle positivo, albendazol na concentração de $0,5 \%$, diferentemente dos ovos que integraram o controle negativo, impossibilitou a abertura dos opérculos dos ovos, qualificando-os como inviáveis. O efeito do albendazol é bem caracterizado por sua classe, os benzimidazóis. Os extratos de $0,1 \%$ (Figura 1 D) $0,25 \%$ (Figura 1 E) e $0,5 \%$ (Figura $1 \mathrm{~F}$ ) obtiveram ação sobre os ovos. O uso do extrato da casca do fruto de $P$. granatum $L$. na concentração de $0,1 \%$ não comprometeu a abertura do opérculo (Figura $1 \mathrm{D}$ ), porém, não foi observada formação miracidial no interior do ovo, podendo ser ainda verificada alterações de superfície, como espessamento de cápsula. 
A concentração de $0,25 \%$ não alterou o padrão morfológico externo do ovo, mas pode-se observar abertura de opérculo e visualização de miracídios em seu interior, sugerindo ação na eclosão dos miracídios (Figura $1 \mathrm{E}$ ), a de $0,5 \%$ (Figura 1 F) não foi observada abertura de opérculo, apresentou dobraduras na casca do ovo e sem presença de miracídio. Resultados similares ao espessamento apresentado nas concentrações de $0,1 \%$ e $0,5 \%$ foram relatados por Taha et al. (2014), que verificaram espessamento dos envoltórios dos ovos de Fasciola gigantica quando expostos a ação de hipoclorito de sódio.

A concentração de $0,5 \%$ do extrato de $P$. granatum comprometeu diretamente o processo de embriogênese dos ovos de $F$. hepatica, tornando-os inviáveis (Figura 1 F). Ademais, alterações no padrão morfológico, descritas na ação da concentração de $0,25 \%$, não foram demonstradas. Tais resultados corroboram com aqueles descritos por Fairweather et al. (2012) quando avaliaram a influência dos benzimidazóis na eclodibilidade dos ovos de $F$. gigantica. $O$ resultado apresentado pode indicar uma resposta do ovo frente ao estresse químico, tanto no caso do albendazol $0,5 \%$ e nos momentos dos extratos nas concentrações de $0,1 \%$ e $0,5 \%$.

A avaliação da atividade fasciolicida dos extratos sobre os adultos foi realizada por observação dos grupos experimentais, com auxílio de um estereomicroscópio, ao longo de $0 \mathrm{~h}, 3 \mathrm{~h}, 12 \mathrm{~h}$ e $15 \mathrm{~h}$ de exposição, podendo visualizar a morte (ou não) dos parasitos. Além disso, foi possível observar alterações macroscópicas visíveis a olho nu dos exemplares analisados, tais como alterações de bordas, turgência, consistência e liberação de conteúdo dos parasitos.

Quando comparado a relação do tempo com as diferentes concentrações do extrato de $P$. granatum L. utilizadas, observou-se que na avaliação de uma hora os extratos analisados não diferiram entre si (Tabela 2). Após três horas de exposição, apenas o extrato na concentração de $0,5 \%$ demonstrou atividade fasciolicida, diferindo significativamente dos demais grupos. Já nos períodos de 12 e 15 horas de exposição, todas as concentrações do extrato analisadas, bem como o albendazole apresentaram eficácia fasciolicida satisfatória.

TABELA 2. Média de motilidade de Fasciola hepatica sob ação do extrato vegetal da casca do fruto de Punica granatum $L$. testado nas concentrações de $0,1 \%, 0,25 \%$ e $0,5 \%$ após ensaio fasciolicida.

\begin{tabular}{lcccc}
\hline $\begin{array}{l}\text { Extrato } \\
\text { vegetal }\end{array}$ & HORA 1 & HORA 3 & HORA 12 & HORA 15 \\
\hline Extrato 0,1\% & $3.00000 \mathrm{a}$ & $1.00000 \mathrm{ab}$ & $0.00000 \mathrm{~b}$ & $0.00000 \mathrm{~b}$ \\
& & & & \\
$\begin{array}{l}\text { Extrato } \\
0,25 \%\end{array}$ & $3.00000 \mathrm{a}$ & $1.00000 \mathrm{ab}$ & $0.00000 \mathrm{~b}$ & $0.00000 \mathrm{~b}$ \\
Extrato 0,5\% & $3.00000 \mathrm{a}$ & $0.00000 \mathrm{~b}$ & $0.00000 \mathrm{~b}$ & $0.00000 \mathrm{~b}$ \\
\hline
\end{tabular}




\begin{tabular}{lllll}
\hline $\begin{array}{l}\text { Albendazol } \\
0,5 \%\end{array}$ & & & & \\
RPMI & $2.33333^{\underline{a}}$ & $2.33333^{\underline{a}}$ & $\begin{array}{l}3.000 \\
00 \mathrm{a}\end{array}$ & $3.00000 \mathrm{a}$ \\
& & & & \\
$\mathrm{P}=$ & 0,1705 & 0,0113 & $<.000$ & $<.0001$ \\
& & & 1 & \\
\hline
\end{tabular}

Médias dos dados após teste de normalidade, seguidas por mesma letra minúscula na coluna, não diferem entre si pelo teste de Tukey $(p<0,05)$;

$\mathrm{Na}$ avaliação histológica dos adultos de $F$. hepatica os achados histológicos dos espécimes tratados com o extrato da casca do fruto de $P$. granatum $\mathrm{L}$. nas concentrações de $0,1 \%$ e $0,5 \%$ (Figuras 2 D e 2 F) foram similares quando comparados com aqueles tratados com albendazol a $0,5 \%$ (Figura $2 \mathrm{C}$ ), sendo estes principalmente caracterizados no comprometimento da formação ultra-estrutural dos espinhos no tegumento de $F$. hepatica, diferindo dos exemplares não tratados (meio $\mathrm{RPMI}$ ), como demonstrado nas figuras $2 \mathrm{~A}$ e B. Por outro lado, na concentração de $0,25 \%$ não foram observadas alterações estruturais na superfície externa do tegumento de F. hepatica.

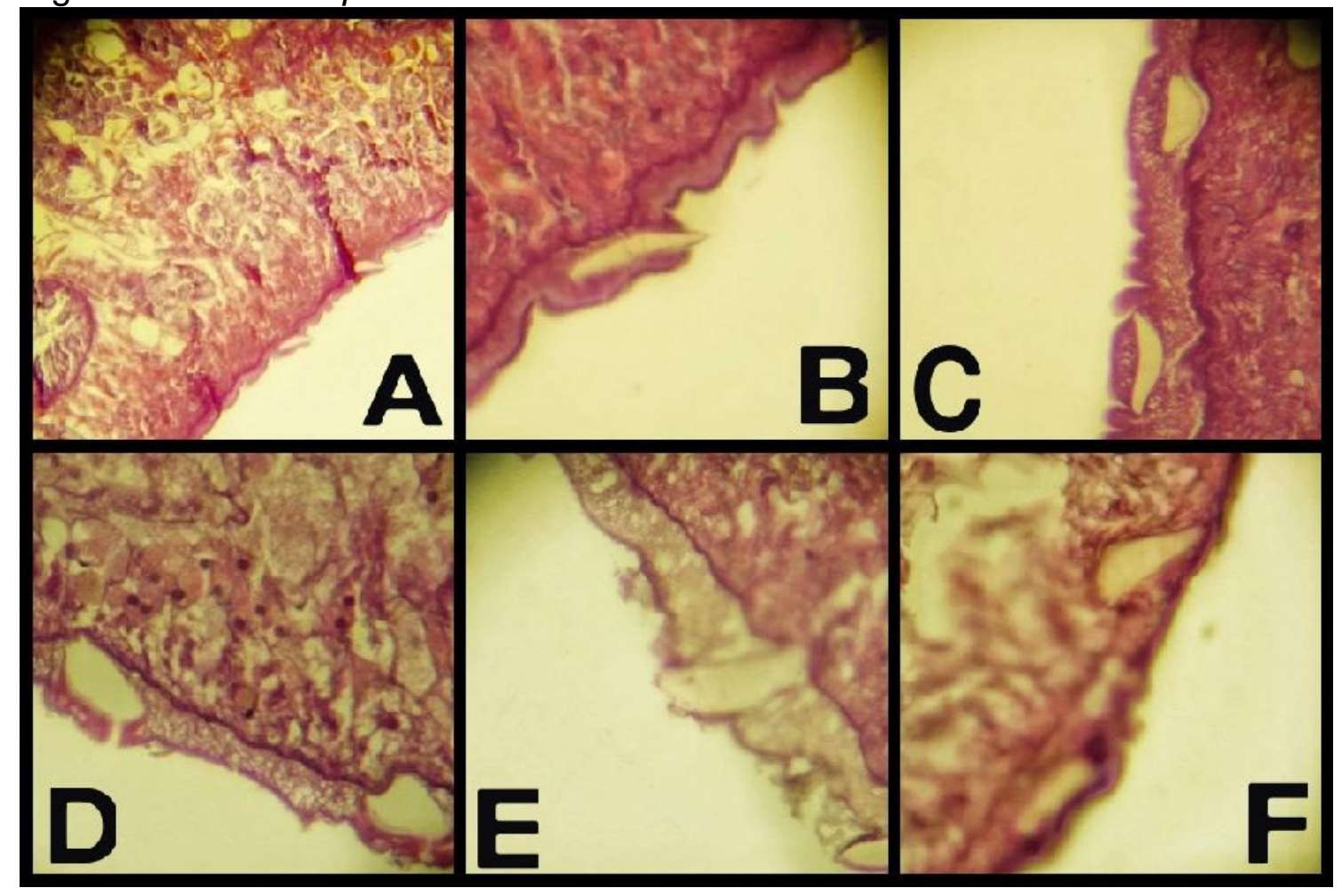

FIGURA 2. Fotomicrografia de alterações histológicas na superfície externa do tegumento de Fasciola hepatica submetidos ao teste. A e B, meio RPMI- com aumento de 10x e 40x, respectivamente, em destaque os espinhos da membrana externa do tegumento; C, exemplar submetido ao albendazol 0,5\% em aumento de 40x; D,E e G exemplares sob ação do extrato da casca do fruto de Punica granatum L. na concentração de $0,1 \%, 0,25 \%$ e $0,5 \%$, respectivamente, em aumento de $40 x$. FONTE: VIDAL, (2017). 
Nas avaliações realizadas, observaram-se alterações no tegumento que de acordo com Lopez-Velez et al. (1999), alterações no tegumento de F. hepatica expostas a ação do triclabendazol, demonstraram inibição na síntese de espinhos em toda a extensão do helminto. Segundo tais autores, a metabolização do triclabendazol resulta na formação do triclabendazol-sulfoxido e otriclabendazolsulfona, os quais inibem a síntese da ultraestrutura nos tegumentos de $F$. hepatica. Assim, mecanismos similares podem estar ocorrendo no presente estudo, justificando as alterações histológicas na superfície externa do tegumento dos espécimes expostos aos extratos de $P$. granatum e ao albendazol. Na concentração de $0,1 \%$ (Figura 2 D) e 0,5\% (Figura 2 E) as alterações foram semelhantes ao albendazole, com retração de espinhos, já na de $0,25 \%$ a retração foi mais leve, deixando os espinhos pouco encapsulados.

\section{CONCLUSÕES}

A partir dos resultados obtidos com o presente estudo é possível concluir que o extrato apresentou atividade em todas as concentrações nos ovos e nos adultos principalmente na concentração de $0,5 \%$, sendo inclusive superior àquela demonstrada pelo albendazol $0,5 \%$ após três horas de análises. $O$ potencial fasciolicida constatado pelo extrato de $P$. granatum pode ser justificado pela presença dos taninos, flavonóides e compostos fenólicos presentes no extrato após análise fitoquímica.

\section{AGRADECIMENTOS}

O presente trabalho foi realizado com apoio da Coordenação de Aperfeiçoamento de Pessoal de Nível Superior - Brasil (CAPES 001), Universidade Federal do Espírito Santo e Fundação de Amparo à Pesquisa e Inovação do Espírito Santo (FAPES).

\section{REFERÊNCIAS}

ALVAREZ-MERCADO, J.M.; IBARRA-VELARDE, F.; ALONSO-DÍAZA, M.A.; VERAMONTENEGRO, Y.; AVILA- ACEVEDO, J.G.; et al. In vitro antihelmintic effect of fifteen tropical plant extracts on excysted flukes of Fasciola hepatica. BMC Veterinary Research, v.11, n.45, p. 1-12, 2015. Disponível em: https://www.ncbi.nlm.nih.gov/pubmed/25890066 doi: https://doi.org/10.1186/s12917015-0362-4

ANDREANI, L.H.; LEMFERS, T.R.; FAUSTINO, F.P.; NOGUEIRA, C.E.; LUZ, T.V.B. Descarte de fígados bovinos por fasciolíase hepatica em Santa Catarina, Brasil. Revista CFMV, v.13 n.73, p.66-71, 2017.

COLES, G.C.; STAFFORD, K.A. Activity of oxyclozzanide, nitroxynil, clorsulon and albendazole against adult triclabendazole resistant Fasciola hepatica. Veterinary Record, v. 148, n.23, p. 723-724, 2001. Disponível em: https://www.ncbi.nlm.nih.gov/pubmed/11430684

doi:

http://dx.doi.org/10.1136/vr.148.23.723.

FAIRWEATHER, I.; MCSHANE, D.D.; SHAW, L.; ELLISON, S.E.; O'HAGAN, N.T.; et al. Development of an egg hatch assa for the diagnosis of triclabendazole resistance in Fasciola hepatica: Proof of concept. 
Veterinary Parasitology, v. 183 n.3, p. 249-259, 2012. Disponível em: https://www.ncbi.nlm.nih.gov/pubmed/21821359 doi: http://dx.doi.org/10.1016/j.vetpar.2011.07.023.

JACOBS, D; FOX, M.; GIBBONS, L.; HERMOSILLA, C. Platyhelminthes ('flatworms'). In: JACOBS, D.; FOX, M.; GIBBONS, L.; HERMOSILLA, C. Principles of Veterinary Parasitology, 1. ed. Oxford, UK: Wiley-Blackwell, . cap. 5, p. 285356. v. 1, 2015.

LALHMINGCHHUANMAWII, K.; VEERAKUMARI, L.; RAMAN, M. Anthelmintic activity of Punica granatum L. ethanol extract against paramphistomes in infected sheep. Journal of Research in Animal Sciences, v.2, n.1, p 79-86, 2014. Disponível em: https://www.researchgate.net/publication/275965419_Anthelmintic_activity_of_Punic a_granatum_ethanol_extract_against_paramphistomes_in_infected_sheep

LOPEZ-VELEZ, R.; DOMINGUEZ, C.A.; GARRÓN, C. Successful treatment of human fascioliasis with triclabendazole. European Journal Clinical Microbiology infection Disease,18 (7): 525-526, 1999. Disponível em: https://www.ncbi.nlm.nih.gov/pubmed/10482035

MATOS, F.J.A. Introdução a fitoquímica experimental. 3 ed. Fortaleza-CE: Edições UFC, p. 14, 2009.

MENEZES, S.M.S.; PINTO, D.M.; CORDEIRO, L.N. Atividades biológicas in vitro de Punica granatum L. (romã). Revista Brasileira de Medicina, v.65, n.11, p. 388-391, 2008. Disponível em: http://pesquisa.bvs.br/brasil/resource/pt/lil-505726

NASCIMENTO JUNIOR, B.J.; SANTOS, A.M.T.; SOUZA, A.T.; SANTOS, E.O.; XAVIER, M.R.; et al. Estudo da ação da romã (Punica granatum L.) na cicatrização de úlceras induzidas por queimadura em dorso de língua de ratos Wistar (Rattus norvegicus). Revista Brasileira de Plantas Medicinais, Campinas, v.18, n.2, p.423432, 2016. Disponível em: http://www.scielo.br/scielo.php?pid=S1516$05722016000200423 \&$ script=sci_abstract\&tlng $=$ pt

doi: http://dx.doi.org/10.1590/1983-084X/15_125

RINALDI, G.; MORALES, M.E.; ALREFAEI, Y.N.; CANCELA, M.; CASTILLO, E.; et al. RNA interference targeting leucine aminopeptidase block shatcdohing of Schistosoma mansoni eggs. Molecular and biochemical parasitology, v.167, n. 2, p.118-126, 2009.2 Disponível em: https://www.sciencedirect.com/science/article/pii/S0166685109001418 . doi: http://dx.doi.org/10.1016/j.molbiopara.2009.05.002

TAHA, H.A.; EL-SHAIKH, K.A.; AL-SADI, M.M. Effect of sodium hypochlorite on Fasciola gigantica eggs an the intermediate host, Lymnaea natalensis: A scanning electron microscopy study. Journal of Taibah University for Science, v. 8, n. 2, p. 75-83, 2014. Disponível em: https://www.tandfonline.com/doi/abs/10.1016/j.jtusci.2013.12.003 doi: https://doi.org/10.1016/j.jusci.2013.12.003 
TUNHOLI, V.M.; LORENZONI, P.O.; DA SILVA, Y.H.; TUNHOLI-ALVES, V.M.; BOELONI, J.N.; et al. Molluscicidal potential of Heterorhabtidis baujardi (Rhabditida: Heterorhabditidae), strain LPP7, on Lymnaea columela (Gastropoda: Pulmonata): An alternative for biological controlo f fasciolosis. Journal Acta Trópica, v. 173, pp. 2329, 2017. Disponível em: https://www.ncbi.nlm.nih.gov/pubmed/28545895 doi: http://dx.doi.org/10.1016/j.actatropica.2017.05.024

QUEVEDO, L.S.; BRUHN, F.R.P.; TEIXEIRA, J.L.R.; ALBERTI, T.S.; SCHEID, H.V.; et al. Epidemiological and clinical-pathological aspects of fasciolosis in livers of cattle slaughtered in southern Brazil. Pesquisa Veterinária Brasileira, v.38 n.9, p.17611766, 2018. Disponível em: http://www.scielo.br/pdf/pvb/v38n9/1678-5150-pvb-3809-1761.pdf doi: http://dx.doi.org/10.1590/1678-5150-pvb-5880. 